\title{
Treatment of Localized Retinal Re- detachment in Silicon Oil Filled Eyes
}

\author{
Muhammad Tariq Khan, Sidrah Riaz, Qasim Lateef Chaudhry
}

Pak J Ophthalmol 2019, Vol. 35, No. 2

See end of article for authors affiliations

Correspondence to: Muhammad Tariq Khan (MBBS, FCPS)

Associate Professor Ophthalmology

Akhter Saeed Medical and Dental College

Bahria Town, Lahore

Farooq Hospital/Medicare Eye Center

E-mail: stariq69@hotmail.com
Purpose: To assess the success rate of retinal reattachment surgery in localized re-detachment in Silicon oil filled eyes which had previously undergone primary retinal detachment (RD) repair with pars plana vitrectomy (PPV) with silicon oil.

Study Design: Retrospective cross sectional case series.

Place and Duration: Farooq Hospital and Medicare eye centre, Lahore from October 2016 to November 2017.

Material and Methods: All those patients were included in our study who underwent primary retinal attachment surgery with pars plana vitrectomy (PPV) and silicon oil for complicated retinal detachment as an internal tamponade and later presented with localized inferior retinal detachment within 6 months of previous RD repair. Patients with total detachment, extensive PVR, retinal shortening, gas tamponade, total $\mathrm{Re} \mathrm{RD}$, and external scleral buckle were excluded. Two ports were made under local anesthesia instead of three conventional ports, simple non irrigation vitrectomy technique was used to achieve retinal reattachment under silicon oil without exchange of SO.

Results: Ten eyes of ten patients were included, eight (8) males and two (2) females. The age range was from 18 to 79 years. Most common cause of re detachment (RD) in all cases was proliferative retinopathy (PVR) followed by retinal break. The new retinal break was identifiable in three (3) cases. All cases attained anatomical success in term of complete retinal attachment after second operation in one year follow up period.

Conclusion: Simple non irrigation vitrectomy surgical technique under local anesthesia is effective, economical and time saving as compared to complicated three port vitrectomy with oil exchange.

Keywords: Silicon oil, rhegmatogenous retinal detachment, proliferative retinopathy, retinal break, Perflourocarbon.
$\mathrm{R}$ etinal detachment (RD) is separation of neurosensory retina (NSR) from retinal pigment epithelium (RPE). Incidence of rhegmatogenous retinal detachment (RRD) repair surgery is 6.3 to 17.9 per 100,000 population annually. First surgical repair attempt fails to attain anatomical success in 10 to $20 \%$ cases and needs a second surgery and $5 \%$ cases are unsuccessful even after second surgical repair ${ }^{1}$. In eyes with proliferative retinopathy (PVR) success rate of anatomical repair are less ${ }^{2}$. Major risk factors for developing rhegmatogenous retinal detachment (RRD) are myopia, lattice degeneration, intraocular surgery aphakia or pseudophakia and Nd: YAG capsulotomy. Silicon oil
(SO) is a good long term intraocular tamponade used in repair of RRD and retinal detachment (RD) associated with $\mathrm{PVR}^{2-5}$. It is a good choice in complicated retinal detachments ${ }^{11-15}$ but when used as tamponade, it is also associated with certain complications like corneal band keratopathy, high intraocular pressure (IOP), lens opacification, hypotony and possible retinal toxicity ${ }^{6}$. Although the current techniques of retinal repair are much improved, still PVR is the most common cause of redetachment. PVR can occur even in eyes filled with SO as endotamponade, mostly in inferior quadrant ${ }^{7}$. The major causes of failure of first attachment repair are missed breaks, anterior or posterior PVR, poor patient 
head positioning, inadequate endolaser application and retinal shortening. The proliferative retinopathy (PVR) is a clinical syndrome associated with retinal traction and detachment in which cells with proliferative potential contract and there is multiplication on the retinal surface and in vitreous $\mathrm{s}^{8-11}$. Some degree of PVR is found in up to $10 \%$ of $\mathrm{RD}^{12-4}$. It takes 4 to 12 weeks usually to develop PVR. There are multiple techniques of retinal re-detachment repair with $\mathrm{SO}$ in situ i.e. surgical intervention with or without silicon oil removal, elimination of membranes with or without retinectomy, use of PFCL with internal tamponade of gas or silicon oil. The purpose of our study was to assess the success rate of retinal reattachment surgery for localized re-detachment under oil in eyes which had previously undergone primary retinal detachment (RD) repair with pars plana vitrectomy (PPV) with silicon oil with simple two ports non irrigation technique without SO exchange.

\section{MATERIAL AND METHODS}

There were 10 eyes of 10 patients included in the study who presented with re-detachment in eyes filled with silicon oil. All patients had history of RD repair with PPV and SO (primary vitrectomy) within the last 6 months. Anatomical success rate was defined as complete reattachment of retina and functional success rate was defined as recovery of ambulatory vision that was counting fingers (CF) or above.

All patients presented within 6 weeks of first RD repair. The primary $\mathrm{RD}$ repair was performed somewhere else in nine (9) patients by different eye surgeons and one was operated by same surgeon who performed all secondary surgeries. All second surgeries in ten (10) patients were performed by single surgeon in Medicare eye centre, from November 2016 to October 2017. Inferior retinal re-detachment with grade C 1 PVR was observed in all cases under silicon oil. All patients were delayed till 6 to 8 weeks after primary RD repair surgery to allow membranes to mature so that membrane could be removed easily as grabbing of immature membranes during surgery can be difficult.

Scleral buckling was not used in any of these cases. Seventy percent cases showed PVR in one quadrant of retina (in seven patients) while open new breaks were identified in $30 \%$ cases (in three patients). None of these cases showed opening of primary break.
All patients underwent thorough history, clinical examination on slit lamp with wide field indirect fundus lens and indirect ophthalmoscope. On history poor post operative positioning was common among all patients. Visual acuity (VA), pupil reaction, IOP (intra ocular pressure) was noted and fundus diagram was drawn to show any identifiable break and extent of retinal detachment. The grading of PVR was done according to Retina society classification $1983^{15}$. Patients were examined postoperatively at day one than at 1 st, 3 rd and $6^{\text {th }}$ weeks and then two monthly.

Under local anesthesia two sclerectomies were created at 2 and 11 o'clock positions with 23 gauge. One port was used for endoillumination and the other for second instrument (laser probe, flute needle and cannula). The vitreous cutter was not used in our procedure and silicon oil was not removed. Membranes were peeled off from surface of retina with retinal forceps and scissors. As all patients had inferior RD, inferonasal retinotomy was created with help of cautery and subretinal fluid (SRF) was aspirated under silicon oil through flute needle. Laser photocoagulation was applied to the entire previously detached retina sparing the macula. Laser application extended from ora serrata to inferior vascular arcade. To counteract hypotony more Silicon oil was injected if required during surgery. Sclerectomy ports were closed.

\section{RESULTS}

There were 8 male patients and 2 female patients (Fig. 1).

The age range was 18 to 79 years with a mean of $49 \pm 30$ years. Proliferative retinopathy (PVR) was the

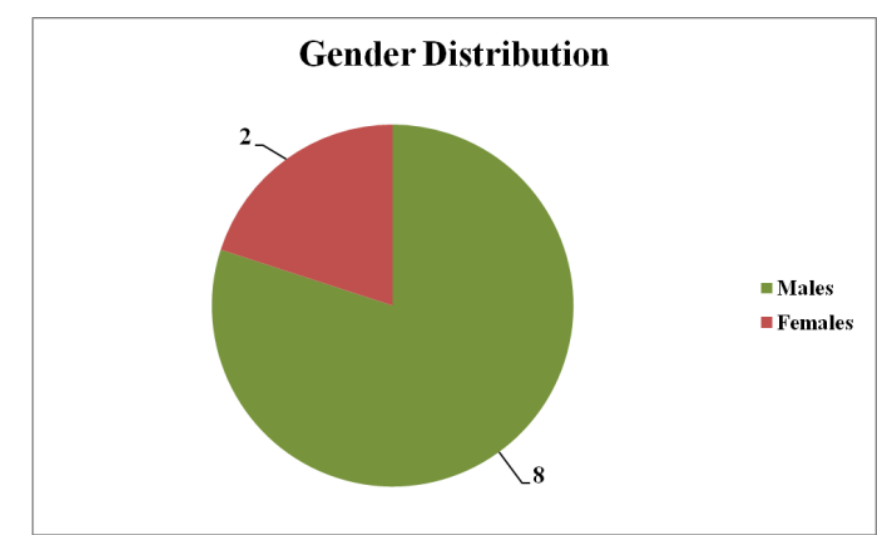

Fig. 1: 
leading cause of re-detachment due to inadequate positioning, incomplete oil fill and inadequate laser. Retinal breaks were seen in 30\% cases. In $70 \%$ cases break was not found (Fig. 2).

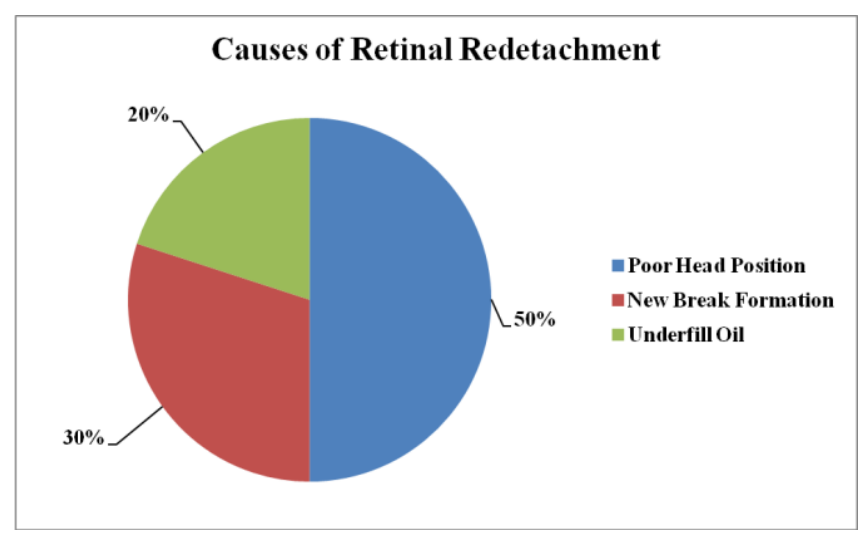

Fig. 2:

Nine patients were pseudophakic and 1 was aphakic. As silicon oil was not removed therefore the total time duration of the procedure was reduced considerably. All the patients were followed up for one year and all patients were stable with ambulatory vision (counting fingers or above) with anatomically successful attachment. None of these patients suffered from glaucoma, corneal opacification, band keratopathy or oil emulsification after one year of follow up. All patients had silicon oil in situ as tamponade and silicon oil was not removed till 6 months of follow-up.

Table 1: Showing cause of Re-RD.

\begin{tabular}{|llccc|}
\hline \multirow{2}{*}{ Sr. } & Causes & \multicolumn{2}{c|}{ No. of Patients } & \% \\
& & Male & Female & age \\
\hline 1. & Poor Head Position & 4 & 1 & 50 \\
2. & New Break formation & 3 & 0 & 30 \\
3. & Under Fill Oil & 1 & 1 & 20 \\
& $\quad$ Total & 8 & 2 & 100 \\
\hline
\end{tabular}

\section{DISCUSSION}

After primary repair of complicated retinal detachment with silicon oil16-18, recurrent detachment may still occur due to open retinal break with or without PVR ${ }^{19-22}$. The rate of recurrence of $\mathrm{RD}$ in $\mathrm{SO}$ filled eyes varies from $21.4 \%$ to $77 \%{ }^{25}$. Recurrence of $\mathrm{RD}$ under $\mathrm{SO}$ provides management challenge. Unfortunately, guidelines for the diagnosis and management of these complicated cases are not defined clearly.

Multiple factors contribute towards failure of primary retinal detachment (RD) repair. There are multiple options available for surgery under SO including membrane surgery with SO in situ, followed by removal of membranes and internal tamponade with SO or gas, and supplementing with SB without repeat vitrectomy. The major causes of re-detachment after first repair are missed breaks during primary repair (small peripheral breaks located at vitreous base are difficult to identify during PPV so 360 laser photocoagulation is recommended). Perisilicon oil proliferation due to poor patient head positioning is seen in early post operative period of incomplete oil fill. Even with complete fill a small concave meniscus of vitreous fluid remains inferiorly when patient is upright and oil bubble rises slightly superiorly. This vitreous fluid contains inflammatory and metaplastic cells and proteins leading to proliferation on retinal surface in 50 to $60 \%$ eyes called perisilicon proliferation ${ }^{23}$. Incidence of macular pucker in eyes with PVR is 5 to $15 \%$ and peeling is not difficult under silicon oil. Second surgery was delayed till 2 months to allow membranes to mature. Membranes under the SO may vary in thickness and adherence to the underlying detached retina. Sometimes they are so thin, pigmented, and strongly adherent to the retina that it is difficult to get at an edge and lift. More often, these membranes in SO-filled eyes can be held and removed with intraocular forceps with great ease. As the retina remains attached under oil, membrane removal is easier. Surgery under oil has an advantage in reducing the operation time. There are different techniques to treat re-detachment including complete redo vitrectomy after removal of silicon oil, use of segmental or encircling scleral buckle with external drainage of SRF; silicon oil may or may not be injected. Gas may be used to reinforce internal tamponade. We used a simple technique without any scleral buckle and found it effective for achieving anatomical success during 12 months follow up period. No statistical data is available on such study in Pakistan but results of our study are comparable with studies in other

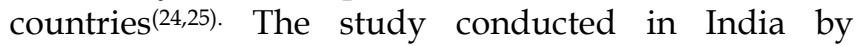
Nagpal et al. showed success rate of $85.2 \%{ }^{25}$ and in our study success rate was $100 \%$ in selected patients. It is recommended to use 5000 CS silicon oil as its complications are less than 1000 CS silicon oil.

The limitation of our study was that the sample 
size was small and more extensive studies are required to elaborate the results further.

\section{CONCLUSION}

The results of this small case series showed that irrigation free vitrectomy with added laser photocoagulation without scleral buckling is effective in term of achieving retinal attachment. This is significant time saving technique as silicon oil is not removed for treatment of inferior re-detachment in eyes filled with silicon oil with without extensive proliferation.

\section{FINANCIAL DISCLOSURE}

The authors have no financial interest.

\section{Author's Affiliation}

Dr. Muhammad Tariq Khan

MBBS, FCPS

Associate Professor Ophthalmology

Akhter Saeed Medical and Dental College

Bahria Town Lahore

Dr. Sidrah Riaz

MBBS, DOMS, FCPS, FRCS

Associate Professor Ophthalmology, Akhter Saeed

Medical and Dental College, Bahria Town, Lahore

Dr. Qasim Lateef chaudary

MBBS, FCPS, FRCS

Associate Professor of Ophthalmology

Jinnah Hospital, Lahore

\section{Author's Contribution}

Dr. Muhahammad Tariq Khan

Concept, study design, primary surgeon, critical analysis.

\section{Dr. Sidrah Riaz}

Data collection, Manuscript writing, assistant in surgery.

Dr. Qasim Lateef chaudary

Critical analysis.

\section{REFERENCES}

1. Cibis PA, Becker B, Okun E, Canaan S. The use of liquid silicone in retinal detachment surgery. Arch Ophthalmol. 1962; 68: 590-9.

2. Scott JD. The treatment of massive vitreous retraction by the separation of pre-retinal membranes using liquid silicone. Mod Probl Ophthalmol. 1975; 15: 285-90.

3. Watzke RC. Silicone retinopoiesis for retinal detachment: a long-term clinical evaluation. Arch Ophthalmol. 1967; 77: 185-96.

4. Cockerham W, Schepens CL, Freeman HM. Silicone injection in retinal detachment. Mod Probl Ophthalmol. 1969; 8: 525-40.

5. Leaver PK, Grey RHB, Garner A. Silicone oil injection in the treatment of massive preretinal retraction: II. Late complications in 93 eyes. Br J Ophthalmol. 1979; 63: 3617.

6. Gonvers M, hornug JP, de Couten C. The effect of liquid silicon on the rabbit retina. Arch Ophalmol. 104; 1057-62

7. Singh AK, Glaser BM, Lemor $\mathbf{M}$, et al. Gravitydependent distribution of retinal pigment epithelial cells dispersed into the vitreous cavity. Retina. 1986; 6: 77-80.

8. Scott JD. Treatment of massive vitreous retraction. Trans Ophthlmo Soc UK. 1975; 95: 429-32.

9. Machemer R. pathogenesis and classification of massive periretinal proliferation. Br J Ophthalmol. 1978; 62: 73747.

10. The classification of retinal detachment Proliferative vitreoretinopathy. Ophthalmology, 1983; 90: 121-5.

11. Machemer R, Aaberg TM, Freeman HM, et al. An updated classification of retinal detachment with Proliferative vitreoretinopathy. Am J Ophthalmol. 1991; 112: 159-65.

12. Charteris DG, Sethi CS, Lewis GP, et al. Proliferative vitreoretinopathy developments in adjunctive treatment and retinal pathology. Eye (Lond). 2002; 16: 369-74.

13. Tseng W, Cortez RT, Ramirez G, et al. Prevalence and risk factors for proliferative vitreoretinopathy in eyes with rhegmatogenous retinal detachment but no previous vitreoretinal surgery. Am J Ophthalmol. 2004; 137: 1105-15.

14. Heimann $\mathbf{H}$, Bartz-Schmidt $\mathrm{KU}$, Bomfeld $\mathbf{N}$, et al. Scleral buckling versus primary vitrectomy in rhegmatogenous retinal detachment: a prospective randomized multicenter clinical study. Ophthalmology, 2007; 114: 2142-54.

15. The Retina society Terminology committee. The classification of retinal detachment with PVR. Ophthalmology, 1983; 90: 121-125.

16. Cox MS, Trese MT, Murphy PL. Silicone oil for advanced proliferative vitreoretinopathy. Ophthalmology, 1986; 93 (5): 646-650.

17. McCuen 2nd BW, Landers 3rd MB, Machemer R. The use of silicone oil following failed vitrectomy for retinal detachment with advanced proliferative vitreoretinopathy. Ophthalmology, 1985; 92 (8): 1029_ 1034.

18. Grey RH, Leaver PK. Results of silicone oil injection in massive preretinal retraction. Trans Ophthalmol Soc UK. 1977; 7 (2): 238 - 241. 
19. McCuen 2nd BW, de Juan Jr E, Landers 3rd MB, Machemer R. Silicone oil in vitreoretinal surgery. Part 2: results and complications. Retina. 1985; 5 (4): 198-205.

20. Federman JL, Schubert HD. Complications associated with the use of silicone oil in 150 eyes after retinavitreous surgery. Ophthalmology, 1988; 95 (7): 870-876.

21. Sell $\mathrm{CH}$, McCuen 2nd BW, Landers 3rd MB, Machemer R. Long-term results of successful vitrectomy with silicone oil for advanced proliferative vitreoretinopathy. Am J Ophthalmol. 1987; 103 (1): 2428.

22. Yang CM. Surgical treatment for diabetic retinopathy: 5- year experience. J Formos Med Assoc. 1998; 97 (7): 477484.

23. Lewis H, Burke JM, Abrams GW, et al. Perisilicone proliferation after vitrectomy for proliferative vitreoretinopathy. Ophthalmology, 1988; 95: 583-91.

24. Yang CM, Hsieh YT, Yang CH, Liu KR. Irrigation-free vitreoretinal surgery for recurrent retinal detachment in silicone oil-filled eyes. Eye (Lond) 2006; 20: 1379-82.

25. Nagpal. M, Chaudhary P., Wachasundar P., Eltayib A., and Raihan A. Indian Journal of Ophthalmology. 2018 Dec; 66 (12): 1763-1771. 